\title{
New high statistics results from the MINERvA experiment Medium Energy run
}

\author{
Heidi Schellman for the MINERvA collaboration ${ }^{a, *}$ \\ ${ }^{a}$ Department of Physics, Oregon State University and Fermilab \\ 301 Weniger Hall, Corvallis, OR, 97333 USA \\ E-mail: Heidi.Schellman@oregonstate.edu
}

The MINERvA experiment has completed its physics run using the $6 \mathrm{GeV}$, on-axis NuMI ME beam at Fermilab. The experiment received a total of $12 \times 10^{20}$ protons on target in both neutrino and antineutrino mode running. This allows MINERvA a new level of statistics in neutrino interaction measurements with the ability to measure multi-dimensional differential cross sections. In addition, in order to make the most of this jump in statistics, a new level of precision in flux prediction has been achieved. We present results from MINERvA's Medium Energy (ME) physics program, including the new kinematic regimes that are now accessible.

40th International Conference on High Energy physics - ICHEP2020

July 28 - August 6, 2020

Prague, Czech Republic (virtual meeting)

${ }^{*}$ Speaker 
MINERvA is a muon neutrino scattering experiment that ran at Fermi National Accelerator Laboratory (Fermilab) between 2010 and 2019 in the NuMI beamline [1] at Fermilab. The main goal of the experiment is precision measurements of neutrino scattering cross sections in the energgy range 2-30 GeV on a suite of nuclear targets, including Helium, Carbon, Water, Iron and Lead. The MINERvA detector is described in detail in [2]. The active detection compenents are triangular scintillating bars mounted in hexagonal frames. Nuclear targets are mounted at the front of the detector, with a central "tracker" region with fiducial mass 5.3 tonnes consisting of active scintillator alone, followed by an electromagnetic calorimeter (ECAL) made up of interleaved lead and scintillator layers for electromagnetic shower detection and an hadronic calorimeter (HCAL) consisting of iron and scintillator layers to detect any residual hadronic energy. Muons with energies above $1.5 \mathrm{GeV}$ are momentum analyzed in the magnetized iron-scintillator MINOS near detector which lies 2 meters beyond the last MINERvA detector plane. Figure 1 shows an event display of a $v+n \rightarrow \mu^{+}+p$ candidate interaction in the MINERvA detector.

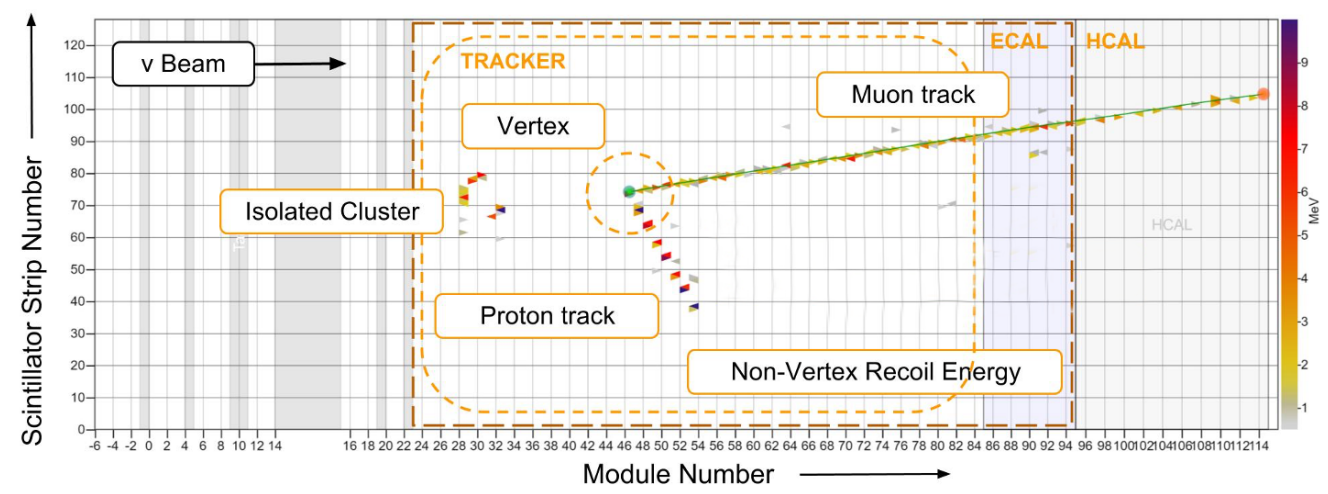

Figure 1: An event display of a $v+n \rightarrow \mu^{+}+p$ candidate quasi-elastic scatter in the MINERvA experiment. Nuclear targets are shown as greyed areas on the left and the ECAL and HCAL are on the right. The $\mu^{+}$ candidate leaves the MINERvA detector and is momentum analyzed in the (unshown) MINOS near detector.

The NuMI neutrino beam ran at two different energies, first, from 2010-2012 in a Low Energy (LE) configuration optimized for on-axis experiments and after 2013 in a Medium Energy (ME) configuration optimized for the NOvA off-axis experiment. The beamline was tuned to focus positive or negative charged particles resulting in enhanced muon neutrino or anti-neutrino content. Figure 2 shows the relative neutrino flux for the LE and ME running. The peak neutrino energy in the LE running was around $3.5 \mathrm{GeV}$ while for the $\mathrm{ME}$ running the peak energy was around $6 \mathrm{GeV}$.

The neutrino fluxes shown in Figure 2 are first estimated using a detailed simulation of the NuMI beamline[3] with additional improvements provided by a reweighting procedure [4] that takes into account hadronic cross sections in the target region and the beamline geometry and magnetic fields. For the LE running this yielded an overall uncertainty on the muon neutrino fluxes of around $8 \%$ [5]. The statistics available in the ME MINERvA data sample include over 1000 candidate $v_{\mu}+e \rightarrow v_{\mu}+e$ scatters. The cross section for this pure electro-weak process is very well know and can be used as a $\sim 3.9 \%$ constraint on the total neutrino flux. This work is presented in reference [6].

The ME data sample includes 825,000 quasi-elastic muon neutrino candidates. MINERvA measures a Charged Current Quasielastic-like (CCQE-like) observable which includes quasi-elastic 

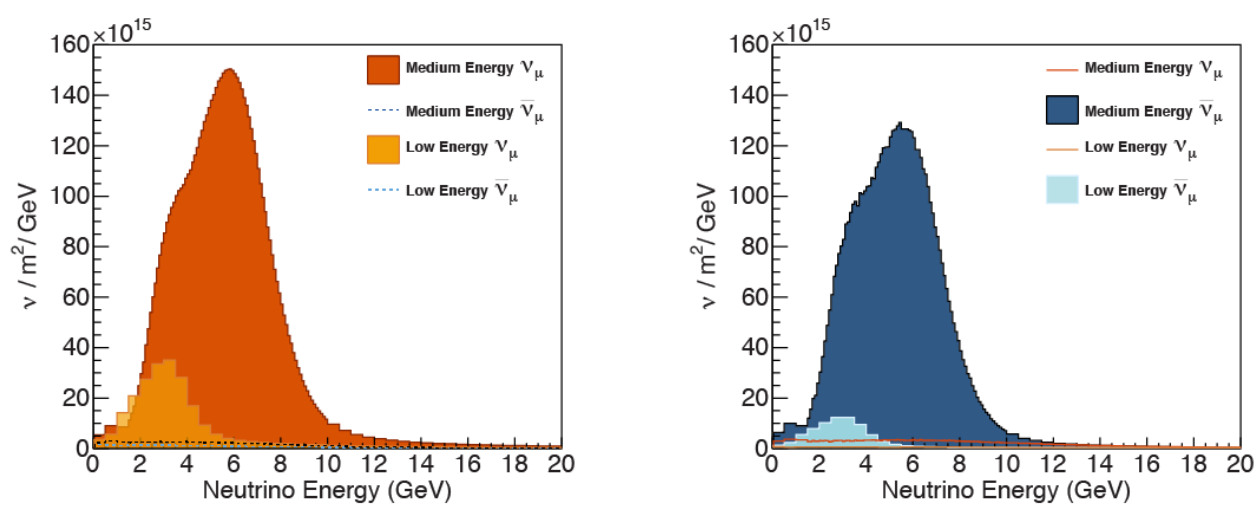

Figure 2: Estimated neutrino flux as a function of neutrino energy for the (left) neutrino tune and the (right) antineutrino tune. Both the LE and ME fluxes are shown.

scatters from multi-nucleon states by allowing any number of nucleons in the final state but no pions. The 2-dimensional CCQE-like scattering cross section as a function of muon kinematics $\left(p_{\|}, p_{\perp}\right)$, as presented in reference [7], is shown in Figure 3. The data are compared to a GENIE[8] 2.12.6 model which includes MINERvA tunes to final state interactions. For the MINERvA v1 tune presented here, modifications include screening of CCQE scattering at low momentum transfer, $Q$, modeled by an Random Phase Approximation (RPA), suppression of non-resonant pion production as suggested by bubble-chamber data[9] and a version of the Valencia[10,11] 2p2h model that has been enhanced to fit MINERvA LE data. See [9, 11, 12] for the model inputs. Figure 5 illustrates a preliminary high-statistics study of the visible hadronic energy in bins of muon $p_{\perp}$ and $p_{\|}$which will allow further enhancements to models for neutrino scattering in the few $\mathrm{GeV}$ range.

Figure 6 shows the cross section as a function of $Q_{Q E}^{2}$, the momentum-transfer squared, calculated from the muon kinematics assuming a target at rest. The data are compared to default GENIE, variations of the MINERvA v1 tune and to the NuWro[14] and GiBUU[15] models.

Analysis of the quasi-elastic channel for anti-neutrinos is being finalized. 477,000 candidates are available, as compared to 13,000 in the LE anti-neutrino sample published in 2018[13]. Figure 4 shows preliminary background subtracted event numbers as a function of muon momentum kinematic variables compared to the tuned MINERvA v1 model described above. The functional form of the data and general agreement with the simulation are similar to those for the neutrino data. We anticipate publication of these data by the end of 2020 .

Many other studies with the full ME data sample are underway, including nuclear cross section ratios, inclusive scattering and studies of exclusive final states with pions and kaons. Our goal is to complete analysis of all of these data in time for ICHEP2022. 


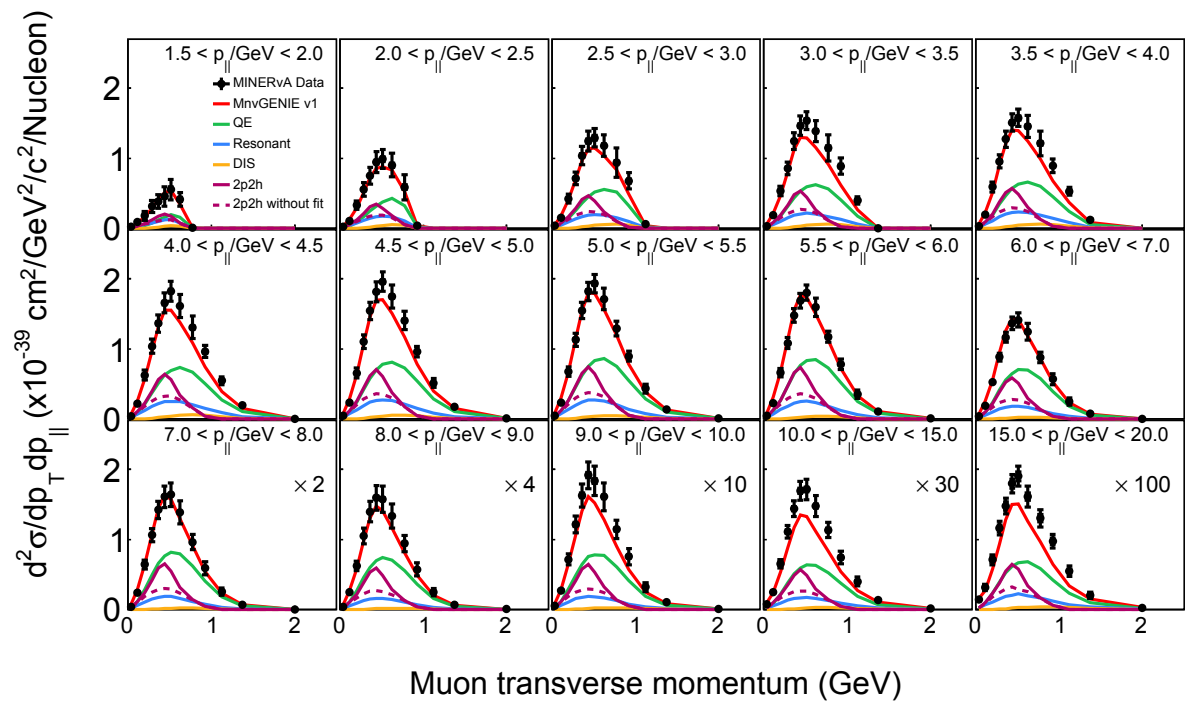

Figure 3: Double Differential quasi-elastic -like scattering cross section for muon neutrino scattering. The curves show the different components that make up the MINERvA GENIE Tune. The solid red curve is the total model prediction, the green curve represents true quasi-elastic scatters within the nucleus, the maroon $(2 \mathrm{p} 2 \mathrm{~h})$ curve shows quasi-elastic scatters from correlated nucleons, the blue and yellow curves show resonant and deep-inelastic scatters that are have a CCQE-like signature due to nuclear scattering and absorption. The dashed lines show the default Valencia 2p2h model while the solid curves show the $2 \mathrm{p} 2 \mathrm{~h}$ model tuned to MINERvA LE data.

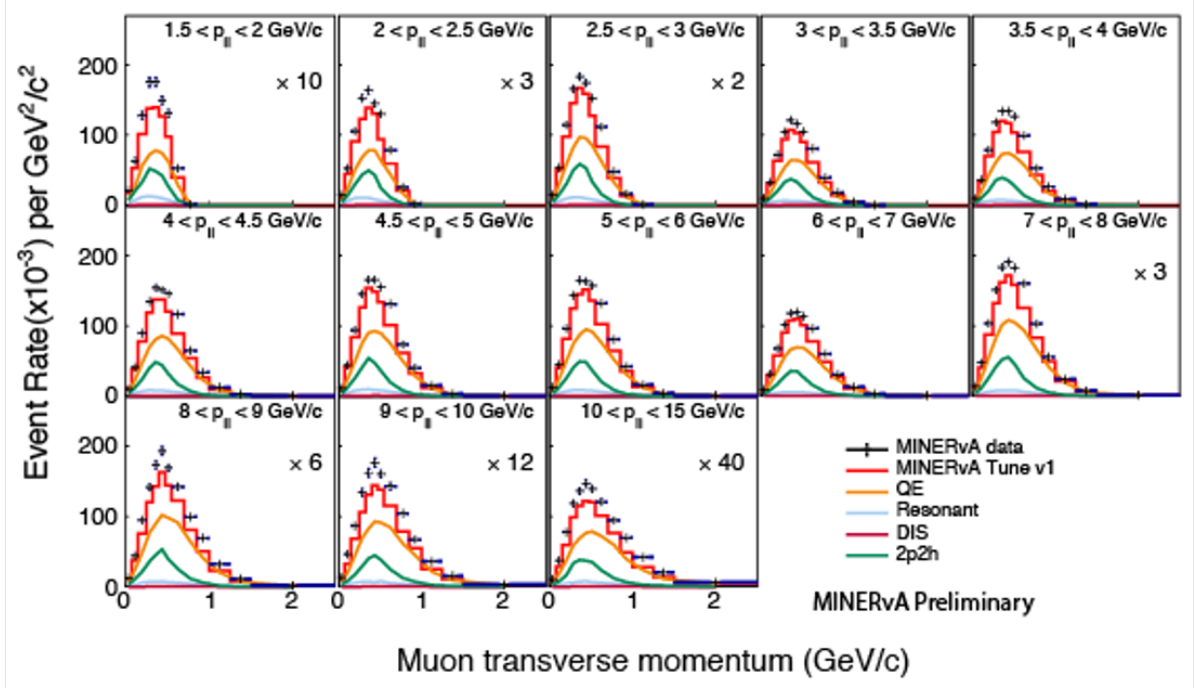

Figure 4: Background-subtracted double differential quasi-elastic-like scattering rates for muon anti-neutrino scattering. The curves show the different components that make up the MINERvA GENIE Tune. The solid red curve is the total model prediction, the orange curve represents true quasi-elastic scatters within the nucleus, the green $(2 \mathrm{p} 2 \mathrm{~h})$ curve shows quasi-elastic scatters from correlated nucleons, the blue and red curves show resonant and deep-inelastic scatters that are have a CCQE-like signature due to nuclear scattering and absorption. 


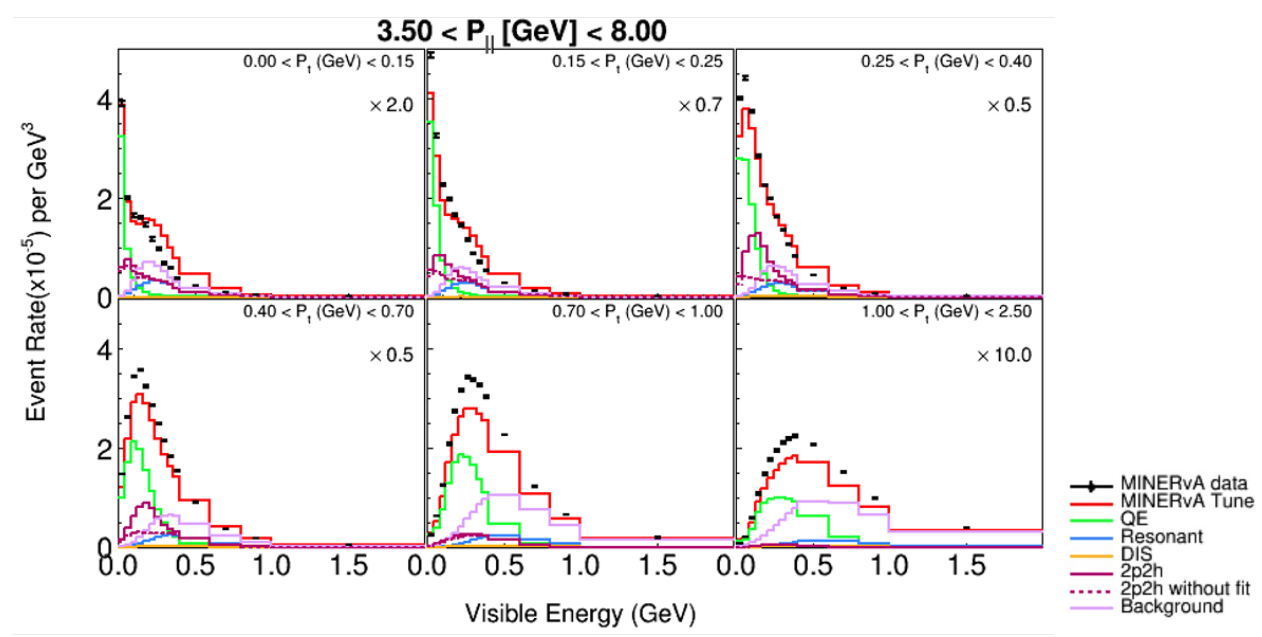

Figure 5: Preliminary study of the triple differential cross section for neutrino scattering as a function of visible energy $E=\sum T_{\text {proton }}$ compared to the models describe above. Backgrounds (purple) due to pion production have not been removed.
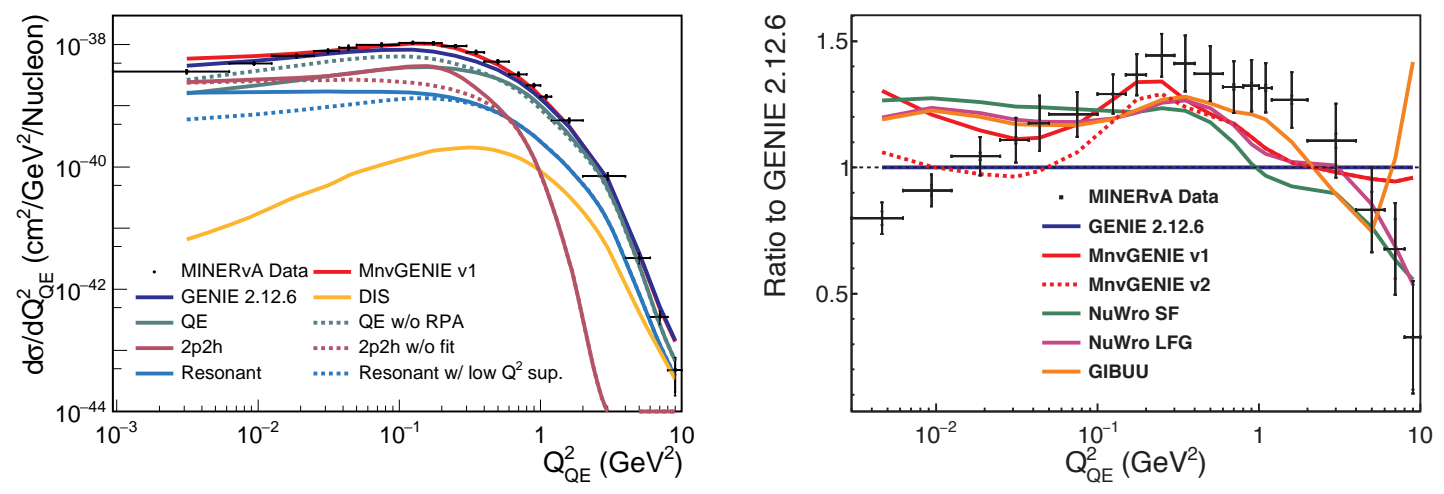

Figure 6: The differential CCQE-like muon neutrino scattering cross section as a function of the 4-momentum transfer squared, $Q^{2}$. The figure on the left shows the cross section with cross section component predictions from various models. The solid lines indicate the components of the MINERvA v1 model, as used in Figure 3 while the dashed lines show modified quasi-elastic without RPA suppression (green dashes), without $2 \mathrm{p} 2 \mathrm{~h}$ tuning (dashed maroon) and without low $Q^{2}$ suppression of pion production. The figure on the right show the ratio to an untuned GENIE 2.12.6 model. Two MINERvA tunes (v1 (red) and v2 (dashed red) ) are shown, as are predictions from the NuWro Spectral and Local Fermi Gas models, and the GiBUU prediction. 


\section{References}

[1] P. Adamson et al., Nucl. Instrum. Meth. A 806, 279 (2016), arXiv:1507.06690 .

[2] L. Aliaga et al. (MINERvA), Nucl. Instrum. Meth. A 743, 130 (2014), arXiv:1305.5199 [physics.ins-det] .

[3] The g4numi software, available at https://cdcvs.fnal.gov/redmine/projects/numi-beamsim/wiki/G4numi.

[4] L. Aliaga et al. (MINERvA Collaboration), Phys. Rev. D94, 092005 (2016), arXiv:1607.00704 [hep-ex] .

[5] L. Aliaga et al. (MINERvA), Phys. Rev. D 94, 092005 (2016), [Addendum: Phys.Rev.D 95, 039903 (2017)], arXiv:1607.00704 [hep-ex] .

[6] E. Valencia, D. Jena, N. Fnu., et al. (MINERvA Collaboration), Phys. Rev. D 100, 092001 (2019).

[7] M. Carneiro et al. (MINERvA), Phys. Rev. Lett. 124, 121801 (2020), arXiv:1912.09890 [hep-ex] .

[8] C. Andreopoulos, C. Barry, S. Dytman, H. Gallagher, T. Golan, R. Hatcher, G. Perdue, and J. Yarba, The GENIE Neutrino Monte Carlo Generator: Physics and User Manual (2015), arXiv:1510.05494 [hep-ph] .

[9] C. Wilkinson, P. Rodrigues, S. Cartwright, L. Thompson, and K. McFarland, Phys. Rev. D 90, 112017 (2014), arXiv:1411.4482 [hep-ex] .

[10] J. Nieves, I. R. Simo, and M. J. V. Vacas, Phys. Rev. C 83, 045501 (2011).

[11] R. Gran, J. Nieves, F. Sanchez, and M. J. V. Vacas, Phys. Rev. D 88, 113007 (2013).

[12] P. Rodrigues et al. (MINERvA), Phys. Rev. Lett. 116, 071802 (2016), [Addendum: Phys.Rev.Lett. 121, 209902 (2018)], arXiv:1511.05944 [hep-ex] .

[13] C. Patrick et al. (MINERvA), Phys. Rev. D 97, 052002 (2018), arXiv:1801.01197 [hep-ex] .

[14] T. Golan, C. Juszczak, and J. T. Sobczyk, Phys. Rev. C 86, 015505 (2012), arXiv:1202.4197 [nucl-th] .

[15] O. Buss, T. Gaitanos, K. Gallmeister, H. van Hees, M. Kaskulov, O. Lalakulich, A. Larionov, T. Leitner, J. Weil, and U. Mosel, Phys. Rept. 512, 1 (2012), arXiv:1106.1344 [hep-ph] . 